\title{
EDITORIAL
}

\section{Kick start training during the COVID-19 pandemic - Challenges of the sporting community}

\author{
Chathuranga Ranasinghe ${ }^{1,2}$, Ranil Jayawardena ${ }^{1,3}$, Venura Palihawadana ${ }^{4}$
}

\begin{abstract}
The COVID-19 pandemic has rapidly spread across the world with increasing morbidity and mortality, leading to implementation of strict preventive measures. Athletes and the sporting community are currently confined to homes, and sporting events are being postponed with times of uncertainty, where physical, technical, and psychological damage is inevitable. The clinical and immunological behavior of the SARS-CoV-2 virus infection is still being analyzed. Exercise in moderation has positive effects on human immunity and wellbeing. Athletes need to be guided to maintain physical, psychological and nutritional conditioning using available knowledge and opportunities. Practice of national health recommendations when training in the home environment and adapting to the 'new normal' environment will be a challenge. Scientific sporting bodies around the world have recommended well planned stepwise structured processes for the resumption of normal training for the safety of the athletes and the wider community. It is recommended, whenever possible that guidance and strategies are developed using the best available scientific evidence.
\end{abstract}

Keywords: COVID-19, Sports, Training, Exercise, Nutrition, Metal health, Immunity

\section{Introduction}

The COVID-19 pandemic has rapidly spread across the globe with increasing morbidity and mortality [1] where countries are still expecting subsequent waves of increased disease incidence.

\section{${ }^{1}$ Sport and Exercise Medicine Unit and \\ ${ }^{2}$ Department of Allied Health Sciences Faculty of Medicine University of Colombo, Colombo Sri Lanka, ${ }^{3}$ Department of Physiology Faculty of Medicine University of Colombo, Colombo Sri Lanka, ${ }^{4}$ Psychiatry Unit Base Hospital Dambadeniya, Dambadeniya Sri Lanka.}

\section{Correspondence:}

Email:chath_r@med.cmb.ac.lk

https://orcid.org/0000-0002-5874-4331
Worldwide prevention strategies employed are social/physical distancing, hand hygiene and cough etiquette, contact tracing, isolation and quarantine, testing, monitoring and promoting general health of the public while vaccinations are still at research level.

Sports provide more than just entertainment, builds social capital, cohesion among communities and provides opportunity for good health and wellbeing [2]. Sporting population is currently confined to homes and major sporting events are being postponed with times of uncertainty. The postponement of these events, including the Olympic Games recently, shows that the sporting world has an important role in the containment of the pandemic. Training routines of athletes around 
the world are being abruptly interrupted where physical, technical, and psychological damage is inevitable [3]. Reducing training and losing their performance capacity can mean loss of competitiveness when returning to competition. Those who are directly and indirectly involved in sports, including coaches, managers, journalists and importantly the fans are negatively affected in different capacities.

Thus, sports administrators, coaches, sports science/medicine professionals and families of the athletes are challenged to help the athletes deal with some of these relevant aspects during this period. This article aims to alert athletes and relevant stakeholders to the need to maintain a conditioning routine and wellbeing during this period and how to plan return to training when the stay home orders are lifted.

\section{Human immune response to COVID-19}

The immune responses induced by SARSCoV-2 virus infection can be clinically explained in 3 stages, where the initial 2 stages are the asymptomatic incubation period and the symptomatic non-severe stage in which a specific adaptive immune response is required to eliminate the virus [4]. The presence of good pre-existing general health and a favorable genetic background (e.g; majorhistocompatibility-complex antigen loci /HLA) are needed for a good endogenous protective immune response [5]. In the third symptomatic inflammation-driven damaging stage, efforts should be made to suppress inflammation and minimize the damage [5]. Enhancing the immune status by improving general health through increased physical fitness, appropriate nutrition, adequate sleep and improvement of mental health is an important public health intervention at this moment. For athletes maintaining the competitive stage will be of a concern. However, this will be a challenge in the background of physical isolation related anxiety and stress, reduced access to regular food, healthcare and financial constraints and changing socioeconomic and behavioral challenges of the individuals, families and societies.

\section{Immune system, exercise and sport}

The positive impact exercise and sport has on the human immune system is well documented [6]. Although scientific data regarding the effects of exercise on SARSCoV-2 is currently not available, host protective effects of exercise from many viral infections including influenza ("Flu"), rhinovirus ("common cold") and herpesviruses [7] is available. An acute aerobic exercise bout of moderate-to-vigorous intensity of less than an hour is known to redeploy highly active immune cell subtypes between the circulation and peripheral tissues (e.g., mucosal surfaces including respiratory and gut epithelia) leading to increased immune surveillance and strengthening of the innate immune response [7]. The exercise bout improves the antipathogen activity of tissue macrophages in parallel with an enhanced recirculation of immunoglobulins, anti-inflammatory cytokines, neutrophils, immature B cells and leads to a dramatic influx of natural killer (NK) cells, and CD8+ T cells [8]. Similar patterns are also seen in different intensities during moderate resistance exercise training [9]. Furthermore, acute and chronic moderate exercises down-regulate excessive inflammation within the respiratory tract which is mediated through multiple pathways. With daily exercise, these acute changes summate to enhance immune defense activity and improve immune regulation and metabolic health [7] placing athletes at a greater advantage with regard to the defense against viral infections as they continue to exercise and be physical fit compared to the general public. 


\section{Is there too much exercise during this period?}

During periods of vigorous training or competition (e.g. running marathon) exceeding an hour have shown reduction in circulating immune cells in peripheral blood 1-2 hours following the training, and these return to preactivity levels after 24 hours [7]. This phenomenon termed "open-window theory of susceptibility to infections", has been attributed to immune suppression and susceptibility to upper respiratory tract infections (URTI) with the increased doses of intense exercise [8]. However, this hypothesis is now being debated due to unavailability of robust evidence about the dose response relationship [8] and the presence of other factors which reduce the immune competency and can cause an URTI, such as psychological stress and anxiety, nutritional deficiencies, poor hygiene and organism virulence [8]. Since the behavior of the SARS-CoV-2 virus is still not fully understood and most people are confined to challenging environments, it is advisable that general public to adhere to the standard World Health Organization (WHO)/ American College of Sports Medicine (ACSM) recommendations of 150 minutes per week of moderate-intensity aerobic physical activity and 2 sessions per week of muscle strength training [10]. This is an important point to be considered by the athletes as part of the public to have gradual training strategies without sudden excessive high dose training.

\section{Training during and return after the COVID-19 pandemic}

Athletes currently training in the home environment should plan to maintain physical and psychological conditioning with the practice of physical distancing and recommended hygiene practices. Quickly adopting the 'new normal way of living' will be of importance during the resumption of proper training. A stepwise and a gradual process needs to be implemented for the resumption of normal training. When to resume should be decided contextually by the respective governments and health sectors to ensure the safety of athletes and the wider community. While giving specific recommendations to individual sports will be challenging due to the inadequate evidence in the current situation; the Australian Institute of Sport (AIS) [11] and Sri Lanka Sports Medicine Association (SLSMA) has developed graded return to play plans for athletes, both recreational and professional which shows the importance of planned gradual return to training to be followed involving all stakeholders.

\section{Types of training available}

- Elite athletes are ideally involved in strength and conditioning, sport specific skill training, building psychology (build motivation, ambition and confidence), nutritional interventions, improving knowledge of the game and tactical game planning for competition. Some of these essential training is missed at the moment and athletes might have to creatively plan home training according to the available opportunities.

- Athletes who were following regular highintensity high-volume training ( $>2-3$ hours per day) should plan the strength and conditioning program during this period to 'maintain' fitness with periodization and adequate rest in between sessions (high work: rest ratios)[12, 13]. It is advised to limit daily exercise sessions to 60 minutes initially. After prolonging periods of inactivity and sudden increase of the training dose can lead to delayed onset muscle soreness (DOMS) and even more severe injury [14]. National Strength and Conditioning Association

(NSCA) 
recommends resistance training to be limited to 2-3 days per week with adequate rest in between [12]. Good hydration, proper hygiene, adequate sleep and adequate carbohydrate intake will help maintain the immune state in the environment with a virulent virus in place.

- Sport specific skill and technique training is missed by most athletes as they have no access to their teams, venues, equipment and coaches. Creative use of technology, video recording skill techniques and analysis, coupling mental imagery with physical and skill training [15] are areas that athletes and coaches can explore during this time.

- Tactical game planning and analysis can be an area given less priority by the athletes and teams previously. This can be an opportunity to go into detail to reflect on the game, own strengths, weaknesses, opportunities and threats to plan for future competition. Considering the possibility of a quick return to training and then competitions; the teams can plan their training strategies.

\section{Things to consider while exercising}

- While maintaining physical distancing and good hygiene; national health guidelines may/may not state all to wear face masks while training when the stay home orders are lifted. If so, exercising and training with a face mask would be extremely challenging to athletes depending on the type of sport, environment (high humid environments like Sri Lanka which can cause increased sweating) and anxiety of perceived health risks due to the mask.

- There is no scientific evidence available on physiological effects of exercise while wearing face masks currently used by the public during this pandemic. Elevation Training Masks (ETM) have been used by professional athletes for high intensity short duration training (30-45 minutes per session for 6 weeks ) to simulate altitude training and respiratory muscle training [16]. These well-fitting masks are known to allow a multi-level resistance system to breathe. However, in order to simulate altitude, the mask must have a mechanism to decrease partial pressure of oxygen, inducing a hypoxic state during exercise which has been suggested to increase aerobic capacity $\left(\mathrm{VO}_{2} \mathrm{max}\right)$, endurance performance, and lung function. It is assumed that increased respiratory load and decreased ventilation might be associated with psychological discomfort (i.e., perceived breath effort) [17].The masks currently used in prevention of COVID-19 infection are very much different to ETMs. People use different types of facemasks which lack standards, made with different material, thickness, resistance levels and without proper fitting. Using a mask will not cause hypoxia in the same altitude in a healthy person but can give variable resistance to breathing, for which they can get accustomed with gradual use.

- Exercising individually in an open environment using standard hygiene practices, personal gear (water bottle, towel, equipment) and physical distancing is becoming more practical at the current point of time. But when training with a team all above will become challenges. In addition, some sports which need body contact (Rugby, wrestling etc.) and common use of equipment (e.g. balls used in cricket, rugby, basketball and shuttlecocks in badminton). Scientific sporting bodies (e.g. AIS ) have proposed quick 'get in, train and get out' [11] strategy from the venue/session to 
minimize person to person exposure. They also have developed return to play protocols to be used, which has to be put into practice to see the feasibility.

- Indoor stadiums will have closed environments occupied by groups of athletes performing high intensity exercise with increased aerosol generation creating a high-risk environment for spread of infection [18]. It is advised to have more ventilation with free airflow in these venues and the start of activity is still discouraged compared to open environments [11].

- Spread of COVID -19 infection through drinking water and the use of swimming pools is not yet documented [19]. Presence of SARS-CoV-2 in stools and wastewater has recently been reported where a possible faecal-oral transmission of the virus has been raised, but not confirmed [20]. Proper operation, maintenance, and disinfection (e.g., with chlorine and bromine) of water should be followed and the disinfection of frequently touched surfaces and specific venue protocols need to be developed and be strictly followed [19].

- Availability of screening athletes before the start of training (special considerations for para -athletes, athletes with medical conditions and athletes tested positive for COVID-19) availability of medical help [18], education and training on hygiene practices and monitoring, availability of adequate facilities for hand washing and personal hygiene, regular disinfection protocols of the venues/equipment, providing accommodation with physical distancing, and agreed protocols to maintain venues and systems as per the national health recommendations will need to be placed. However, this will become an additional financial and human resource burden to the respective sporting bodies.

\section{Mental health}

\section{Social isolation, anxiety and increased disease} risks

Periods of isolation and confinement away from training, reduced access to daily requirements, financial constraints and the sense of insecurity can lead to psychological stress and anxiety. This may lead to elevated levels of glucocorticoids which will inhibit many critical functions of our immune system, resulting in increased host susceptibility to infections and non-communicable diseases (NCD) [21]. Exercise in general can mitigate the negative effects of stress to maintain immune function, particularly during prolonged periods of isolation and confinement. It has been shown that astronauts in space travel for nearly 6 months with extreme isolation who had a higher pre-flight cardiorespiratory fitness and muscle endurance had a better immune response compared to astronauts who were not fit [22].

\section{Abstinence from sports}

Mental health impact of abstinence from sports can be a major concern for athletes. They usually live a highly regimented lifestyle and training takes up a major part of their daily schedule. When training is taken away, athletes can struggle trying to find meaningful activities to fill the void. Studies have shown that physical training is also a source of inspiration, coping and well-being for many people including athletes [23]. Prolonged abstinence and its psychological effects had been studied in athletes who were forced to abstain from sports due to injury. These studies have shown abstinence and associated uncertainty can have various psychological impacts including depression, anxiety, loss of self-esteem and overall mood irregularity [24, 25]. 
Postponement of events, lack of access to training venues and possible loss of sponsorships or jobs can be main sources of their uncertainty.

Similarly, abstinence from physical activity has been found to have effects similar to withdrawal comparable to individuals with substance (e.g. alcohol, tobacco and drugs) and behavioral addictions [26], especially among athletes engaging in extreme sports. This suggests, there is some degree of compulsiveness to engage in high intensity physical activities in athletes. Inability to engage in these compulsive coping behaviors can have immediate negative effects on their mood such as anhedonia (inability to feel pleasure in normally pleasurable activities), craving and low mood [26]. Although this withdrawal effect is more remarkable among athletes who engage in highly intense sports, it also can occur to some degree in others engaging in low intense sports [27].

\section{Athletes with mental health problems}

Moreover, athletes have a higher propensity to have preexisting mental health problems which can make them more vulnerable to develop mental health issues during times of isolation and stress [28, 29]. To complicate things further, higher prevalence of stigma, low mental health literacy, fear of appearing weak and false ideas regarding masculinity can prevent athletes seeking help [30, 31]. Coaches play a significant role, especially during stressful times by creating a safe environment conducive to discuss mental health struggles [31].

\section{Mental Toughness and Mindfulness}

Nevertheless, 'mental toughness' has been found to serve as a protective factor against development of these adverse consequences [29]. Mental toughness is how effectively athletes deal with stress, pressure and challenge. Therefore, promotion of mental toughness is important to improve resilience during uncertain times. It has the added benefit of improving their performance in tough competitive environments. By investing themselves in improving mental toughness athletes can achieve some sense of control and satisfaction since they can feel that they are still committed to their cause.

A practical advice coaches can give athletes is, asking them to maintain the momentum of their physically active lifestyle by conditioning them and engaging in some activity, although it might not be proper training. This will keep them psychologically prepared for the actual training. Another way they can maintain their motivation is by embarking on new challenges. Being mindful about their worrying thoughts and focusing on what can be changed rather than ruminating on all possible negative outcomes, is another strategy that can be adopted. Mindfulness exercises have shown promising effects on recovery, awareness and focus of athletes [32]. It can be a great source of stress reduction during uncertain and unfocused times for athletes and, can also be used to enhance their performance.

\section{Nutrition}

There are a few nutritional issues that can arise with prolonged locked down periods. Firstly, due to unavailability of a variety of foods, their nutritional status can deteriorate and lead to marginal micronutrient deficiencies especially among high risk categories such as adolescents, sport personals depend on body weight for competition and those who depended on outside catering support. Protein-energy malnutrition and micro nutritional deficiencies may reduce their immunity and performances. Low vitamin A and D levels, and lower serum selenium and zinc values may impair the 
immunity [33]. In the absences of plasma values, it is recommended to supplement suspected micronutrients for a short period. Moreover, it is essential to move to a balanced diet which covers all groups to overcome deficiencies and get other beneficial nutrients such as antioxidants and probiotics.

Another point to consider is that the detraining period can induce an increase in both body mass and body fat. Many professional level sport personals have had changed lifestyles during the lockdown period in both activity and dietary patterns. Changes in body composition, mainly losing muscle mass and increased fat mass may hamper their performance and increase risk of injuries. Personalized dietary advice tailored to training regimens is required for needed athletics.

\section{Conclusions:}

COVID -19 pandemic has affected the global community physically, psychologically an socioeconomically. Effect to the sporting population is well recognised. The measures to mitigate negative effects will be novel, but basics to maintain healthy life will be no different to the general public. Understanding the behaviour of the virus, its effects on immunity and exercise, and how exercise or sport should be used scientifically to maintain health of athletes and wider community is a priority. Coaches and scientific community can help athletes to maintain physical, psychological and nutritional conditioning within the practice of national health recommendations and to adopt 'new normal way of living'. Well planned stepwise structured processes need to be placed in advance for the resumption of normal training. As we move forward during the pandemic, whenever possible, it is crucial that guidance and strategies are developed using the best available evidence.

\section{Conflicts of interest:}

Authors declare no conflicts of interest.

\section{References}

1.Severe Outcomes Among Patients with Coronavirus Disease 2019 (COVID-19) United States, February 12-March 16, 2020. MMWR Morb Mortal Wkly Rep. 2020;69(12):343-6.

2.Schulenkorf N. Sport for Development events and social capital building: a critical analysis of experiences from Sri Lanka. Journal of Sport for Development. 2013;1(1):25-36.

3.Andreato LV, Coimbra DR, Andrade A. Challenges to Athletes During the Home Confinement Caused by the COVID-19 Pandemic. Strength and conditioning journal.

4.Xu T, Jiang $\mathrm{X}$, Denton $\mathrm{D}$, Kumar $\mathrm{S}$. Ecdysone controlled cell and tissue deletion. Cell death and differentiation.27(1):1-14.

5.Shi Y, Wang Y, Shao C, Huang J, Gan J, Huang $X$, et al. COVID-19 infection: the perspectives on immune responses. Cell Death \& Differentiation.

6.Campbell JP, Riddell NE, Burns VE, Turner M, van Zanten JJ, Drayson MT, et al. Acute exercise mobilises CD8+ $\mathrm{T}$ lymphocytes exhibiting an effector-memory phenotype. Brain Behav Immun 2009 Aug;23(6):767-75 doi: 101016/jbbi200902011 Epub 2009 Feb 28.

7.Nieman DC, Wentz LM. The compelling link between physical activity and the body's defense system. Journal of Sport and Health Science. 2019;8(3):201-17.

8.Campbell JP, Turner JE. Debunking the Myth of Exercise-Induced Immune 
Suppression: Redefining the Impact of Exercise on Immunological Health Across the Lifespan. Frontiers in immunology. 2018;9:648-.

9.Freidenreich DJ, Volek JS. Immune responses to resistance exercise. Exerc Immunol Rev. 2012;18:8-41.

10.(ACSM) ACoSM. Staying Active During the Coronavirus Pandemic. March 24, 2020 Exercise is Medicine; 2020.

11.(AIS) AIoS. AIS framework for rebooting sport in a COVID-19 Environment: AIS; May 2020 [Available from: https://ais.gov.au/_data/assets/pdf_file/0006/7 30374/35845_AIS-Framework-for-RebootingSport-Summary.pdf.

12.(NSCA) NSaCA. COVID-19: NSCA Guidance on Safe Return to Training for Athletes: NSCA; 2020 [Available from: https://www.nsca.com/covid-19-return-totraining.

13.Caterisano A, Decker D, Snyder B, Feigenbaum M, Glass R, House $\mathrm{P}$, et al. CSCCa and NSCA Joint Consensus Guidelines for Transition Periods: Safe Return to Training Following Inactivity. Strength \& Conditioning Journal. 2019;41(3).

14.William Braun GS. ACSM information on delayed onset muscle soreness (DOMS)2011. Available from:

https://www.acsm.org/docs/defaultsource/filesf or-resource-library/delayed-onset-musclesoreness-(doms).pdf?sfvrsn=8f430e18_2.

15.Kathleen AM, Sandra EM, Craig RH. Imagery Use in Sport: A Literature Review and Applied Model. The Sport Psychologist. 1999;13(3):245-68.
16.Porcari JP, Probst L, Forrester K, Doberstein S, Foster C, Cress ML, et al. Effect of Wearing the Elevation Training Mask on Aerobic Capacity, Lung Function, and Hematological Variables. J Sports Sci Med. 2016;15(2):379-86.

17.Jung HC, Lee NH, John SD, Lee S. The elevation training mask induces modest hypoxaemia but does not affect heart rate variability during cycling in healthy adults. Biol Sport. 2019;36(2):105-12.

18.Hull JH, Loosemore M, Schwellnus M. Respiratory health in athletes: facing the COVID-19 challenge. Lancet Respir Med. 2020:S2213-600(20)30175-2.

19.(UNICEF) WHOatUNCsF. Water, sanitation, hygiene, and waste management for the COVID-19 virus: interim guidance. 2020. Report No.: CC BY-NC-SA 3.0 IGO Contract No.: WHO/2019-nCoV/IPC_WASH/2020.3.

20.Heller L, Mota CR, Greco DB. COVID-19 faecal-oral transmission: Are we asking the right questions? Science of The Total Environment. 2020;729:138919.

21.Nieman DC, Wentz LM. The compelling link between physical activity and the body's defense system. Journal of sport and health science.8(3):201-17.

22.Agha NH, Mehta SK, Rooney BV, Laughlin MS, Markofski MM, Pierson DL, et al. Exercise as a countermeasure for latent viral reactivation during long duration space flight. The FASEB Journal.34(2):2869-81.

23.John C, Matthew YWK, Scott V, Guy EJF. Who Uses Exercise as a Coping Strategy for Stress? Results From a National Survey of 
Canadians. Journal of Physical Activity and Health. 2014;11(5):908-16.

24.Chan CS, Grossman HY. Psychological Effects of Running Loss on Consistent Runners. Perceptual and Motor Skills. 1988;66(3):875-83.

25.Smith AM, Scott SG, Wiese DM. The Psychological Effects of Sports Injuries Coping. Sports Medicine. 1990;9(6):352-69.

26.Heirene RM, Shearer D, Roderique-Davies G, Mellalieu SD. Addiction in Extreme Sports: An Exploration of Withdrawal States in Rock Climbers. J Behav Addict. 2016;5(2):332-41.

27.Franken IH, Zijlstra C, Muris P. Are nonpharmacological induced rewards related to anhedonia? A study among skydivers. Prog Neuropsychopharmacol Biol Psychiatry. 2006;30(2):297-300.

28.Åkesdotter C, Kenttä G, Eloranta S, Franck J. The prevalence of mental health problems in elite athletes. J Sci Med Sport. 2020;23(4):32935 .

29.Gerber M, Best S, Meerstetter F, Walter M, Ludyga S, Brand S, et al. Effects of stress and mental toughness on burnout and depressive symptoms: A prospective study with young elite athletes.JSciMedSport. 2018;21(12):12005 .

30.Reardon CL, Hainline B, Aron CM, Baron D, Baum AL, Bindra A, et al. Mental health in elite athletes: International Olympic Committee consensus statement (2019). British Journal of Sports Medicine. 2019;53(11):667.

31.Castaldelli-Maia JM, Gallinaro JGdMe, Falcão RS, Gouttebarge V, Hitchcock ME, Hainline B, et al. Mental health symptoms and disorders in elite athletes: a systematic review on cultural influencers and barriers to athletes seeking treatment. British Journal of Sports Medicine. 2019;53(11):707.

32.Zeljka Vidic MSM, Richard Oxhandler. Mindfulness Meditation Intervention with Male Collegiate Soccer Players: Effect on Stress and Various Aspects of Life. The Sport Journal. 2018;21.

33.Jayawardena R, Sooriyaarachchi P, Chourdakis M, Jeewandara C, Ranasinghe P. Enhancing immunity in viral infections, with special emphasis on COVID-19: A review. Diabetes \& Metabolic Syndrome: Clinical Research \& Reviews. 2020;14(4):367-82. 